Rev. High Pressure Sci. Technol., Vol. 7 (1998) 190 192

\title{
Atomic Structure Based on a Relativistic Current- and Spin-Density Functional Theory
}

\author{
M. Higuchi, Y. Onuki*, and A. Hasegawa $\dagger$ \\ Graduate School of Science, Tohoku University, Sendai, 980-77, Japan \\ *Department of Physics, Faculty of Science, Osaka University, Toyonaka, 560, Japan \\ †Department of Physics, Faculty of Science, Niigata University, Niigata, 950-21, Japan
}

\begin{abstract}
A new single-particle equation of the Kohn-Sham-Dirac type is derived from a relativistic current- and spin-density functional theory ( RCSDFT ), and is here applied to the calculations of the atomic structures of the rare-earth elements. Both the relativistic effects and the magnetic effects are taken into account on an equal footing, and the numerical calculation is carried out by modifying the method of Cortona et al. Because of the presence of the effective magnetic field, the degeneracies in all orbits are completely resolved like the Zeeman splittings. Total spin and orbital angular momenta over all the occupied states are shown to agree reasonably well with Hund's rules for the rare-earth ions.

[atomic structure, density functional calculation, orbital moments, spin polarization, Hund's rules]
\end{abstract}

\section{Introduction}

In order to calculate the electronic structure for f-electron compounds where the felectrons are itinerant and responsible to magnetism, a current- and spin-density functional theory of Vignale and Rasolt [1] should be generalized to the relativistic case, i.e., a relativistic current- and spin-density functional theory (RCSDFT). In a single-particle equation of the RCSDFT which is derived for the isolated atoms [2], the magnetic effects which originate from the spin polarization and the orbital current are expressed in a form similar to the Zeeman term in which the spin and the orbital angular momenta couple with an effective magnetic field. The effective magnetic field is determined selfconsistently.

In this paper, a single-particle equation based on the RCSDFT is applied to the calculations of the atomic structures of rare-earth trivalent ions by following the method of Cortona et al. [3]. The effect of the orbital current is taken into account by using the phenomenological method developed by Eriksson et al. [4].

\section{Results and Discussions}

It is more difficult to solve a single-particle equation of RCSDFT than the usual case without the magnetic field, due to the term of the effective magnetic field. Four coupled equations in which two eigenstates with the same orbital angular momentum $l$ are related by the effective magnetic interaction terms must be solved simultaneously unless the case of $l=0$ or $|m|=l+1 / 2$.

First of all, we consider the spin-polarized case in which the interaction of the orbital angular moment with the effective magnetic field is neglected in the RCSDFT and the exchangezorrelation energy is given as a functional in terms of the charge and spin densities $[3,5]$. Within a non-spin-polarized relativistic local-density approximation, the eigenstate of the total angular momentum $j$ has $(2 j+1)$-fold degeneracy, e.g., the eigenvalues of
$4 \mathrm{f}$ electrons for $\mathrm{Ce}^{3+}$ split into two levels corresponding to $j=5 / 2$ and $7 / 2$, which are sixfold and eightfold degenerate, respectively. However, these degeneracies are completely solved just like the Zeeman splitting under the consideration of the spin-polarized effect. Besides the $4 \mathrm{f}$ eigenstates, splittings of the energy levels can be seen in all other orbits as well. Fig.1 shows the $4 f$ eigenstates of $\mathrm{Ce}^{3+}$ from the spin-polarized relativistic localdensity calculation (case $b$ ) and those from the non-spin-polarized relativistic local-density calculation (case a). For $4 \mathrm{f}$ states, the effects of the internal magnetic field are more important than the spin-orbit coupling.

Similar results are obtained for the other rare-earth trivalent ions. The magnitude of the splittings for the $4 \mathrm{f}$ eigenvalues increase as the atomic number becomes larger, because of the increase of the spin densities.

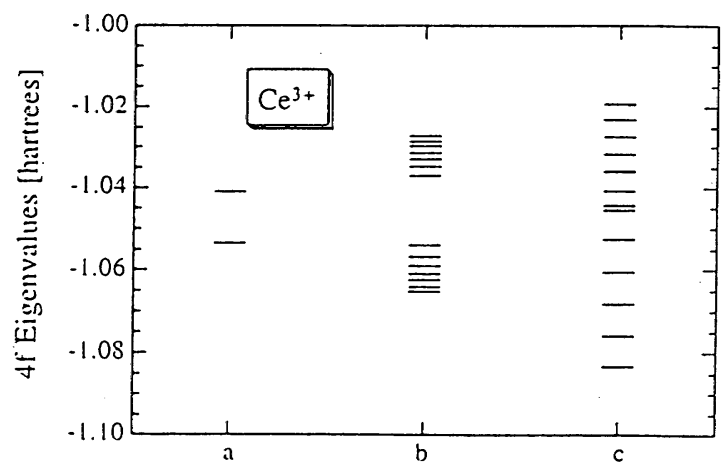

Fig. 1. $4 \mathrm{f}$ eigenvalues of $\mathrm{Ce}^{3+}$ obtained from (a) non-spinpolarized relativistic calculation, (b) spin-polarized relativistic calculation, (c) spin- and orbital-polarized relativistic calculation. 
In Fig. 2, the radial spin densities for the rare-earth trivalent ions are shown. These distributions almost agree with those of the $4 \mathrm{f}$ electron charge density which correspond to the unpaired electrons in the trivalent ions. In Fig.3, the radial spin densities of filled shells for the rare-earth trivalent ions are shown. The spin polarization of filled shells is much smaller than that of the $4 \mathrm{f}$ electron. It is noted that the distribution of the spin density of filled shells is determined by the exchange interaction with the $4 f$ electrons, i.e., in order to gain the exchange energy, the spin of filled shells is aligned in the same direction as the $4 \mathrm{f}$ electrons within the region where the $4 \mathrm{f}$ charge density is considerably large. The effective magnetic field becomes large where the spin polarization appears strongly, as shown in Fig. 4 for $\mathrm{Ce}^{3+}$. These results agree with the results of the relativistic spinpolarized calculation by Cortona et al. [3].

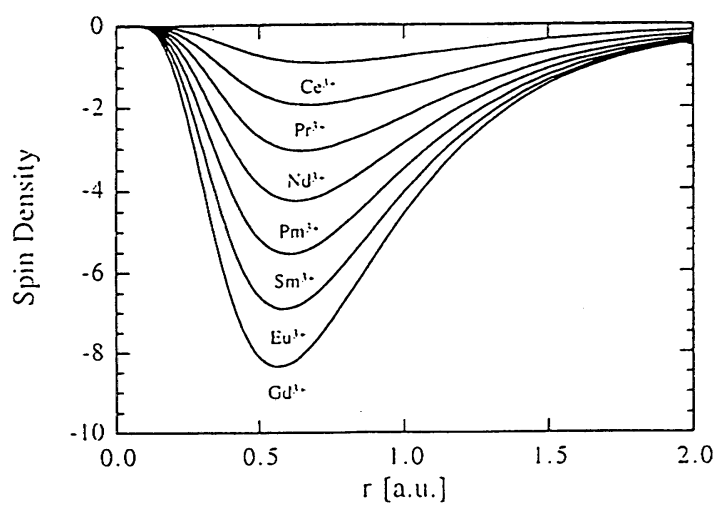

Fig. 2. Radial spin densities for the rare-earth trivalent ions salculated by relativistic spin-polarized density functional theory.

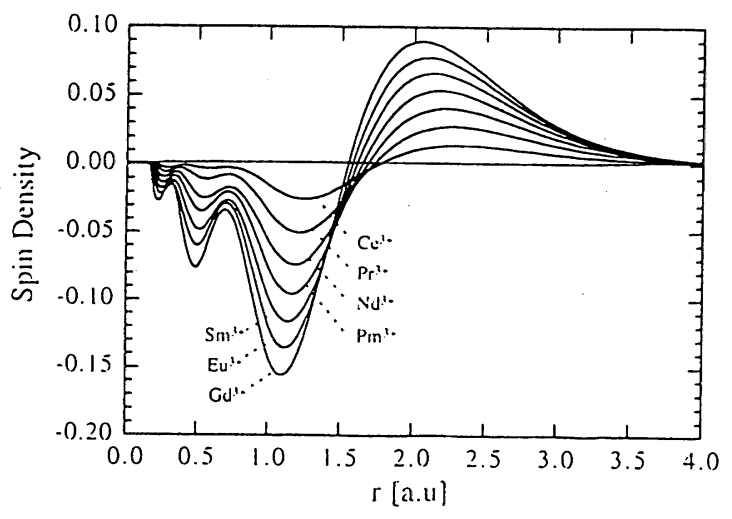

Fig. 3. Radial spin densities of filled shells for the rare-earth trivalent ions calculated by relativistic spin-polarized density functional theory.

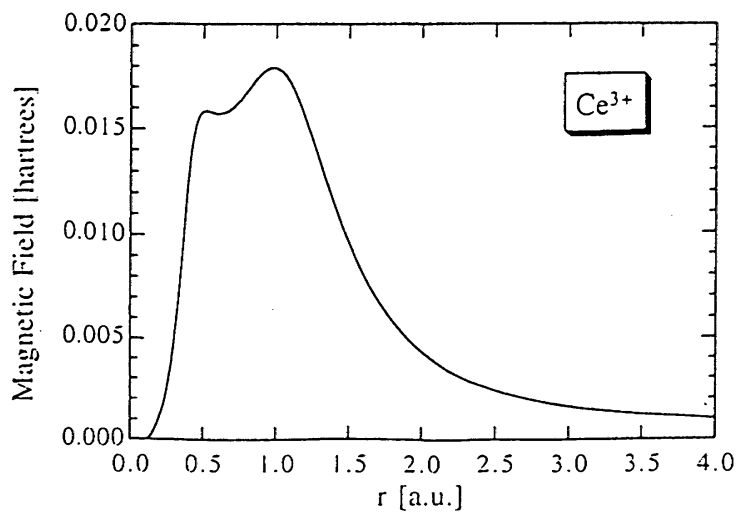

Fig. 4. Effective magnetic field of $\mathrm{Ce}^{3+}$ calculated by relativistic spin-polarized density functional theory.

In order to estimate the effects of the orbital current within the RCSDFT, it is essential to derive the exchange-correlation energy functional which contains the paramagnetic current density as the basic variables in addition to the charge density and spin density. However, such an exchange-correlation energy functional is not available as far as we know. As another sophisticated method where the effects of the orbital current can be taken into account, Eriksson et al. have proposed an additional interaction term arising from the orbital polarization [4]. According to them, the additional term to the spin-polarized Dirac Hamiltonian is given by $-E^{3}<M_{L}>l_{z}$, where $E^{3}$ is the Racah parameter and $\left\langle M_{L}\right\rangle$ is the magnitude of the orbital moment. We recalculated the atomic structures on the basis of the spin-polarized relativistic theory by evaluating $-E^{3}<M_{L}>l_{z}$ as a perturbation. Values of the parameter $E^{3}$ are taken from the experimental $4 \mathrm{f}$ data [6].

The energy corrections caused from the orbital polarization increase as the orbital angular momentum becomes larger, i.e., it is the most remarkable for the $4 \mathrm{f}$ states. In Fig. 1, we compare the $4 \mathrm{f}$ eigenvalues of the spin- and orbital-polarized calculation ( $\operatorname{case}$ ) for $\mathrm{Ce}^{3+}$ with the results of cases (a) and (b). It can be seen that the energy splittings for the $4 \mathrm{f}$ eigenvalues are enhanced in the spin- and orbital-polarized calculation and the effects of the internal magnetic field are more important than the spin-orbit coupling. Similar results are obtained for the other rare-earth trivalent ions though the magnitude of the energy shifts is propotional to $<M_{L}>$.

The spherically averaged total angular, spin angular and orbital angular momentum densities are given by

$$
\begin{aligned}
& M_{:}^{J}(r)=\frac{1}{4 \pi} \int_{\pi \pi} \mathrm{d} \Omega \operatorname{Tr}\left[J_{i} \rho(r)\right], \\
& M_{:}^{\sigma}(r)=\frac{1}{4 \pi} \int_{4 \pi} \mathrm{d} \Omega \operatorname{Tr}\left[\sigma_{:} \rho(r)\right],
\end{aligned}
$$




$$
M_{z}^{\prime}(r)=\frac{1}{4 \pi} \int_{4 \pi} \mathrm{d} \Omega \operatorname{Tr}\left[l_{:} \rho(r)\right]=M_{z}^{J}(r)-\frac{1}{2} M_{z}^{\sigma}(r)
$$

where $\rho(\boldsymbol{r})$ is the density matrix defined as $\rho(\boldsymbol{r})=\sum\left|\Phi_{i}\right\rangle\left\langle\Phi_{i}\right|$, and $\left|\Phi_{i}\right\rangle$ is the eigenstates of a single-particle equation, the sum is taken over all the occupied states. In Fig.5, the total angular, spin angular and orbital angular momentum densities for $\mathrm{Ce}^{3+}$ are shown.

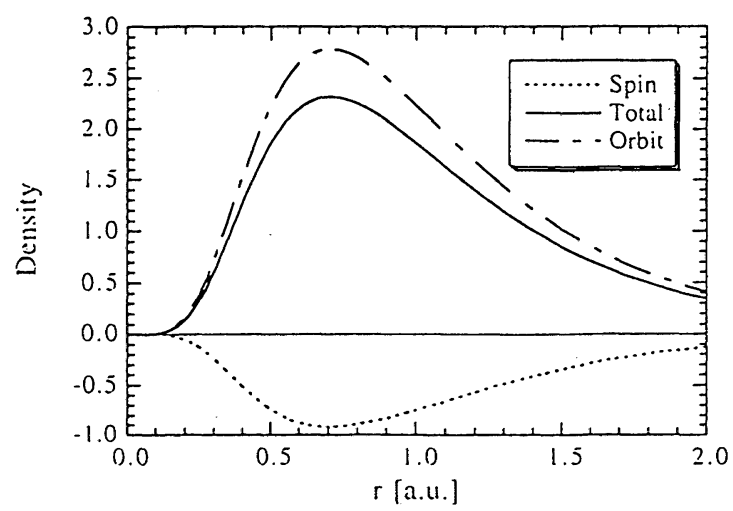

Fig. 5. Radial total angular, spin angular and orbital angular momentum densities of $\mathrm{Ce}^{3+}$ obtained from the spin- and orbitalpolarized relativistic calculation.

Total number of the spin over the occupied states which is obtained by integrating the spin density over the whole region is given in Table 1. It is in good agreement with Hund's first rule, i.e., 0.98 for $\mathrm{Ce}^{3+}, 1.98$ for $\mathrm{Pr}^{3+}$ and 6.97 for $\mathrm{Gd}^{3+}$, etc. are salculated In Table $1,\left\langle M_{s}>/ n_{4 f}\right.$ means the ratio of the number of the aligned spin to the number of $4 \mathrm{f}$ electrons $n_{4 f}$, which presents the degree of the spin alignment. The ratio approaches near toward 1 , as the number of $4 \mathrm{f}$ electrons becomes larger, because the exchange interaction to align the spin grows strong. The sum of the orbital angular momentum over the occupied states is equal to 2.99 for $\mathrm{Ce}^{3+}, \quad 4.99$ for $\mathrm{Pr}^{3+}$ and 0.01 for $\mathrm{Gd}^{3+}$, etc. which are also in good agreement with Hund's second rule. Taking into account the effect of the orbital current by using Eriksson's method, Hund's second rule is satisfied more reasonably, as given by $\left\langle M_{L}\right\rangle_{\text {surrection }}$ in Tablel.

Exchange energy and total energy for the rare-earth trivalent ions are shown in Table 2. The effect of the orbital polarization on the total energy was estimated by $-1 / 2 E^{3}<M_{L}>^{2}$ [4]. It can be seen that the contribution of the orbital polarization to the total energy is comparable to that of the spin polarization, as already shown by Cortona et al. [3].
Table 1. The sum of the spin and orbital angular momentum over the occupied states for the trivalent rare-earth ions.

\begin{tabular}{cccccccc}
\hline & $\mathrm{Ce}^{3+}$ & $\mathrm{Pr}^{3+}$ & $\mathrm{Nd}^{3+}$ & $\mathrm{Pm}^{3+}$ & $\mathrm{Sm}^{3+}$ & $\mathrm{Eu}^{3+}$ & $\mathrm{Gd}^{3+}$ \\
\hline$n_{+i}$ & 1 & 2 & 3 & 4 & 5 & 6 & 7 \\
$\left\langle H_{S}\right\rangle$ & 0.98 & 1.98 & 2.97 & 3.97 & 4.97 & 5.97 & 6.97 \\
$\left\langle H_{S}>h_{+1}\right.$ & 0.981 & 0.989 & 0.992 & 0.993 & 0.994 & 0.995 & 0.996 \\
$\left\langle M_{L}\right\rangle$ & 2.99 & 4.99 & 5.98 & 5.98 & 4.98 & 2.98 & 0.01 \\
$\left\langle M_{L}\right\rangle_{\text {corrction }}$ & 3.00 & 5.00 & 5.99 & 5.99 & 4.99 & 2.98 & 0.01 \\
\hline
\end{tabular}

Table 2. Exchange energy, total energy and the correction to the total energy caused from the orbital polarization for the trivalent rare-earth ions, in hartrees.

\begin{tabular}{llllllll}
\hline & $\mathrm{Ce}^{3+}$ & $\mathrm{Pr}^{3+}$ & $\mathrm{Nd}^{3+}$ & $\mathrm{Pm}^{3+}$ & $\mathrm{Sm}^{3+}$ & $\mathrm{Eu}^{3+}$ & $\mathrm{Gd}^{3+}$ \\
\hline $\begin{array}{c}\text { Exchange } \\
\text { energy }\end{array}$ & -189.80 & -195.39 & -201.10 & -206.93 & -212.88 & -218.96 & .225 .15 \\
$\begin{array}{c}\text { Total } \\
\text { energy }\end{array}$ & $-88+5.24$ & -9221.98 & -9608.58 & -10003.18 & -10411.92 & -10928.92 & -11256.43 \\
$\begin{array}{c}\text { Correction } \\
\text { to total energy }\end{array}$ & -0.0099 & -0.027 & -0.040 & -0.043 & -0.031 & -0.011 & 0 \\
\hline
\end{tabular}

\section{References}

[1] G. Vignale and Mark Rasolt, Phys. Rev. B, 37, 10685 (1988).

[2] M. Higuchi and A. Hasegawa, J. Phys. Soc. Jpn., 6 6, 149 (1997).

[3] P. Cortona, S. Doniach and C. Sommers, Phys. Rev. A, 31 , 2842 (1985).

[4] O. Eriksson, M. S. S. Brooks and B. Johansson, Phys. Rev. $B, 41,7311$ (1990).

[5] A. H. MacDonald, J. Phys. C, 16, 3869 (1983).

[6] L. J. Nugent, J. Inorg. Nucl. Chem., 32, 3485 (1970). 